\title{
Facets of neuroticism and musculoskeletal symptoms. A study of middle-aged twins
}

\author{
Olav Vassend ${ }^{\mathrm{a}}$, Benedicte Humborstad Orvik ${ }^{\mathrm{b}}$, Nikolai Olavi Czajkowski ${ }^{\mathrm{a}, \mathrm{c}}$ \\ and Espen Røysamb ${ }^{\mathrm{a}, \mathrm{c}}$ \\ a) Department of Psychology, University of Oslo, Norway \\ b) Educational Psychology Service, Asker and Barum \\ c) Division of Mental Health, Norwegian Institute of Public Health, Oslo, Norway \\ Correspondence: Olav Vassend, Department of Psychology, University of Oslo, Forskningsveien 3A, NO-0317 Oslo, Norway \\ E-mail: olav.vassend@psykologi.uio.no Telephone: +4722845197
}

\begin{abstract}
Clinical and epidemiological studies have shown that both site-specific and more widespread musculoskeletal (MS) conditions are linked to anxiety and depression symptoms. However, the nature of this relationship is poorly understood, particularly in terms of underlying genetic and environmental influences. Furthermore, the personality trait neuroticism has been shown to be related to common emotional symptoms and somatic distress as well as to more serious psychiatric and medical disorders. In modern personality theory, the broad neuroticism domain is conceptualized as consisting of a set of lower-order facets, such as anxiety, hostility, and depression, which may be differentially related to various health outcome measures. So far, the role of neuroticism facets as risk factors for MS conditions has not been explored in genetically informative designs. In the current study, the relationship between MS symptoms and six neuroticism facets was investigated in bivariate analyses and in regression models including sex, education level, and general health indices as control variables. Using multivariate twin modeling, genetic and environmental influences on the phenotypes and the associations among them were determined. The sample consisted of 746 monozygotic (MZ) and 770 dizygotic (DZ) twins in the age group of 50-65 (mean $=57.11$ years, $\mathrm{SD}=4.5$ ). The results showed that a single factor accounted for about $50 \%$ of the overall variance in MS symptom reporting. Two neuroticism facets, N1: anxiety and N3: depression, appeared as significant in the regression analyses. Both these facets and MS symptoms were strongly influenced by genetic factors [heritability $\left.\left(\mathrm{h}^{2}\right)=0.46-0.54\right]$. While there was a considerable overlap in genetic risk factors between the three phenotypes, a large proportion $(71 \%)$ of the genetic variance in MS symptoms was unique to the phenotype, and not shared with the neuroticism facets.
\end{abstract}

This is an open access article distributed under the Creative Commons Attribution Licence, which permits unrestricted use, distribution, and reproduction in any medium, provided the original work is properly cited.

\section{INTRODUCTION}

Chronic pain has been described as a "silent epidemic" (1) that affects a large proportion of the population, particularly in high-income countries, resulting in great personal suffering and societal costs in terms of sick leave and lost productivity (2). Among the most prevalent chronic or recurrent pain conditions are those affecting the musculoskeletal (MS) system. MS symptoms and disorders encompass pain and other symptoms at specific anatomical sites (e.g., low back pain, shoulder and neck pain), as well as more generalized symptoms as seen in fibromyalgia and chronic widespread pain (CWP). Chronic MS pain affects between $11 \%$ and $50 \%$ of the general population $(2,3)$, with CWP prevalence varying between $11 \%$ and $14 \%$. Such discrepancies in prevalence estimates are partly due to differences in the definition of MS pain and to the methods used for its assessment (2).

Except in cases of traumatic injury and certain diseases affecting MS structures, the etiological factors behind MS conditions are not fully understood and identified $(1,4)$. However, several risk factors have been shown to be associated with disorder onset and development: (a) Demographic factors, e.g., sex, age and education level (1); (b) comorbidities such as sleep disorders, chronic fatigue, irritable bowel syndrome, and mood disorders $(3,5)$; (c) personality traits, in particular neuroticism (6); (d) psychosocial and physical working conditions $(7,8)$; (e) psychological states and strategies that themselves modulate pain experiences, e.g., general anxiety, catastrophizing, fear-avoidance and somatic awareness (9); and (f) genetic factors (1).

Pain problems seem to develop from an early age and tend to persist and worsen by an increasing number of pain sites during later life stages (10). Studies of adolescent populations have shown that MS symptoms are common (11), and emotional and behavioral problems appear to be strongly related to multi-site pain (12). The lifespan nature of MS symptoms and their high prevalence across age groups highlights the need to investigate the genetic and environmental factors underlying such symptoms and their correlates. Twin studies of MS pain generally indicate substantial genetic effects, with heritability estimates ranging from $30 \%$ to $68 \%$ (13). Studies of neck pain have shown that the genetic influence becomes gradually less important with increasing age, and individual-specific (non-shared) environmental factors dominate almost completely in the older age groups $(14,15)$. Of note, in 
populations with similar environmental exposures there is still considerable individual variation in disorder occurrence and severity (4).

Numerous epidemiological and clinical studies have shown that a clear majority of patients with chronic MS pain or other pain conditions are women, and this sex bias appears to correspond to actual sex differences in laboratory pain sensitivity (9). Thus, compared to men women generally have a higher sensitivity and lower tolerance to a range of experimental pain stimuli (e.g., cold and heat pain, muscle pain), in addition to reporting higher pain ratings (9). Furthermore, both functional somatic disorders (e.g., chronic fatigue, irritable bowel syndrome) and internalizing psychiatric disorders (e.g., generalized anxiety disorder, major depression) are more prevalent in women than in men (16), and anxiety and depression frequently occur together with chronic pain in both general population and patient samples $(17,18)$. Importantly, the added burden of depression and anxiety with chronic MS pain is strongly associated with more severe pain, greater disability, and poorer health related quality of life $(19,20)$. Thus, negative affect symptoms, which are related to sex (6), appear to play a crucial role in the development and chronification of MS pain and other pain conditions.

Accumulated research has documented that anxiety/ depression symptoms are moderately to strongly associated with the personality trait neuroticism (21), and twin studies indicate a common genetic predisposition (22). In addition, a couple of twin studies have shown that associations between neuroticism and functional somatic disorders can be attributed to shared genetic and individual-specific environmental factors $(23,24)$. Vassend et al. (25) showed that in a sample of young twins (aged 23-35 years) the relationship between neuroticism and general (non-clinical) somatic complaints could to a large extent be accounted for by a common genetic factor. Moreover, a substantial proportion of the variance in somatic complaints was due to unique genetic and individual-specific environmental influences unrelated to neuroticism. Similar findings have been reported by Hansell et al. (24) in a study of adolescent twins. However, to our knowledge these finding have not been replicated, and it is an open question whether they apply to MS symptoms specifically and to what extent they can be generalized to older individuals.

An additional question, not addressed in previous research in this area, pertains to the facet structure of personality domains and whether the facets or subscales of neuroticism are differentially related to health indices such as MS symptoms. According to Costa and McCrae's five-factor theory of personality $(26,27)$, the neuroticism domain comprises six facets, among them anxiety and depression. In twin studies of the relationship between neuroticism and psychiatric and somatic symptoms $(22,24,28)$, facet information has not been utilized. Although anxiety and depression symptoms are obviously relevant to an adequate understanding of the development and consequences of various pain conditions, it is still an unsettled question which of the neuroticism facets will be most strongly associated with MS symptoms, particularly when adjusting for demographic variables and general health indices.

In the current study, we explored the relationship between MS symptoms and the six neuroticism facets in bivariate analyses and in regression models including sex, education level, and general health indices as control variables. The sample comprised middle aged twins (50-65 years) and were thus older than the participants in the two twin studies of neuroticism and somatic complaints referred to above $(24,25)$. Using a multivariate twin design, we then investigated to what extent common genetic or environmental liability factors contribute to the covariance between neuroticism facets (identified as significant in the regression analyses) and MS symptoms.

\section{METHODS}

\section{Sample}

Twins were recruited from the Norwegian Twin Registry (NTR). The registry comprises several cohorts of twins (29), and the current study is based on a random sample from the cohort born 1945-1960. In 2011, questionnaires were sent to a total of 2136 twins. After reminders, 1516 twins responded, yielding a response rate of $71 \%$. Of the participants, 1272 individuals were pair responders, and 244 were single responders. Zygosity has previously been determined based on questionnaire items and has been shown to classify correctly $97-98 \%$ of the twins (30). The cohort, as registered in the NTR, consists only of same-sex twins, and the study sample consisted of 290 monozygotic (MZ) male twins, 247 dizygotic (DZ) male twins, 456 MZ female twins and $523 \mathrm{DZ}$ female twins. Age range of the sample was 50-65 (mean $=57.11, \mathrm{SD}=4.5)$. The study was approved by the Regional Ethical Committee, and informed consent was obtained from all participants.

\section{Measures}

Neuroticism facets were assessed using a Norwegian version of the NEO Personality Inventory Revised (NEO-PI-R) (26,31). The inventory consists of 240 items and measures five broad domains (i.e., extraversion, neuroticism, agreeableness, openness to experience, and conscientiousness) as well as six facets subsumed under each domain. The neuroticism domain comprises the facets N1: anxiety, N2: hostility, N3: depression, N4: self-consciousness, N5: impulsiveness, and N6: stress vulnerability. Each facet is measured by six items, and Cronbach's alpha for the Norwegian version varies from 0.60 to 0.84 (31).

MS symptoms were measured using the Giessen Symptom Checklist (GSCL) (32,33). This GSCL subscale comprises 6 items, reflecting symptoms at various 
body sites: (1) Pains in joints or limbs, (2) backache, (3) pain in neck and shoulders, (4) headaches, (5) heaviness or tiredness in the legs, and (6) head-pressure. The participants were asked to rate the degree to which they 'generally' suffered from the complaints, using a 5-point scale: 1 - not at all; 2 - slightly; 3 - somewhat; 4 - considerably; and 5 - strongly. In the original development of the scale Brähler and Scheer (32) decided to include additional symptoms (i.e., items 4-6) that are commonly associated with MS pain symptoms proper (i.e., items 1-3). Principal components analysis of the six items yielded only one component with an eigenvalue $>1$ in two sub-samples comprising, respectively, the first and the second member of the twin pair. The first/second eigenvalues in the two sub-samples were $2.92 / 0.95$ and $3.08 / 0.87$, respectively. The factor loadings were in the $0.50-0.80$ range. This common factor explained about $50 \%$ of the variance in MS symptoms in both sub-samples. Chronbach's alpha values for the full and the shortened (items 1-3) scale were 0.80 and 0.76 , respectively, and the correlation between the two scales was $0.93(p<0.001)$. In the present study, in which MS symptoms in the broader sense constitute the main study variable, results based on the original full scale (i.e., the mean of all the 6 measures) will be reported.

As noted, the demographic variables included in the study were sex and education (5 levels). A set of general health indices was adopted from previous largescale Norwegian health surveys $(6,34)$. These were (1) presence of medical disease (yes/no), (2) presence of lasting functional impairment (yes/no), and (3) reduced activity or days in bed due to illness (acute or chronic) or injury for the last two-week period (yes/no), before completing the questionnaire.

\section{Analyses}

Descriptive statistical analyses were performed to examine means (SD) or score distributions of the variables. Bivariate associations between the neuroticism facets and the MS symptoms index were assessed using Pearson correlation analysis. Regression analysis was then performed to examine the independent effects of the neuroticism facets on the MS symptoms index in models including the neuroticism facets alone or together with demographic and general health variables. Because regression analysis needs to reflect the paired structure of the data when the complete sample is examined, Generalized Estimating Equations (GEE) was used (35).

Based on the results from the regression analysis, we conducted a set of biometric analyses to estimate the genetic and environmental contributions to the associations between neuroticism facets and MS symptoms in the best-fitting model. All models were run with OpenMx (36). Standard Cholesky models (37-39) were used to estimate the genetic and environmental contributions to variance in and covariance between the three phenotypes. The Cholesky model specifies as many latent genetic and environmental factors as variables (phenotypes) in a triangular decomposition (for illustration see Figure 1). The estimates of genetic and environmental effects is based on a comparison of resemblance in $\mathrm{MZ}$ twins (who share all segregating genetic material) and DZ twins (who share on average half of their segregating genetic material). Generally, biometric modeling allows for estimating three major sources of variance, i.e., additive genetic factors (A), common environment $(\mathrm{C})$ and individual-specific or non-shared (E) environment. In addition, non-additive genetic effects (D) may be tested, but are only indicated if the observed MZ-correlations are greater than twice the DZ-correlations. Models are constrained so that latent A-factors correlate perfectly among MZtwins, and at 0.5 among DZ-twins. C-factors are correlated at unity for both zygosity groups, and E-factors are by definition uncorrelated. Several nested models were compared in order to identify the best-fitting one according to the minus2LogLikelihood difference test $(\Delta-2 \mathrm{LL})$ and the Akaike Information Criterion (AIC) (40). Thus, an ACE model was compared to an AE model, and the consequences of constraining the parameters to be equal across sex in a given model were assessed. In investigating potentially sex-limited effects of genetic and environmental factors using the Cholesky model, the approach outlined by Neale et al. (39) was adopted. In same-sex twin samples only socalled scalar sex limitation can be assessed, i.e., it is presupposed that the same factors cause variation in males and females, but they may do so to a different extent. Since the factors are the same across sex, they must correlate with each other to the same extent. Hence, the genetic and environmental correlation matrices must be constrained to be equal for males and females. The model with the lowest AIC represents the best balance of goodness-of-fit and parsimony.

\section{RESUlts}

Symptom score distributions and prevalence of symptomatic individuals are shown in Table 1. Pain and other symptoms in the extremities and in the head, neck and back region were reported commonly, with a prevalence of $16.9-60.0 \%$. MS pain symptoms (i.e., items 1-3) were the most prevalent, and a fairly large proportion of the respondents $(8-15 \%)$ indicated that they suffered 'considerably' or 'strongly' from their complaints.

As expected, medical problems were common in this middle-aged twin sample (Table 2). A large number of the respondents $(41 \%)$ reported that they had at least one medical condition, and about $11 \%$ reported having been ill or injured during the last two weeks. All demographic and general health variables were significantly associated with MS symptoms when they were entered simultaneously with the neuroticism facets in regression analysis (Table 2). The phenotypic correlations between the neuroticism facets on the one hand, 
Table 1. Score distributions for the six symptoms included in the MS index, and prevalence of symptomatic individuals (i.e., with a score equal to or greater than 2: Slightly).

\begin{tabular}{lcccccc}
\hline & 1: Not at all & 2: Slightly & 3: Somewhat & 4: Considerably & 5: Strongly & \% symptomatic \\
\hline 1. Pain in joints and limbs & 40.0 & 27.8 & 17.4 & 10.7 & 4.1 & 60.0 \\
2. Backache & 51.4 & 26.4 & 14.0 & 5.9 & 2.3 & 48.6 \\
3. Pain in neck and shoulder & 44.0 & 28.4 & 15.1 & 8.4 & 4.0 & 56.0 \\
4. Headache & 63.4 & 23.6 & 8.8 & 3.3 & 0.9 & 36.6 \\
5. Heaviness/tiredness in legs & 76.3 & 15.2 & 4.8 & 2.6 & 1.1 & 23.7 \\
6. Head-pressure & 83.1 & 11.5 & 3.1 & 1.8 & 0.5 & 16.9 \\
\hline
\end{tabular}

Table 2. Descriptive statistics (second column) and estimates of regression coefficients based on generalized estimating equations, representing statistical effects of neuroticism facets on MS symptoms (third column), and after adjustment for sex, education and general health indices (fourth column). $* * \mathrm{p}<0.01 ; * * \mathrm{p}<0.001$.

\begin{tabular}{lccc}
\hline Variable & $\begin{array}{c}\text { Mean (SD) } \\
\text { /proportions }\end{array}$ & $\begin{array}{c}\text { Regression coefficients: } \\
\text { N facets only }\end{array}$ & $\begin{array}{c}\text { Regression coefficients: } \\
\text { All variables }\end{array}$ \\
\hline N1: Anxiety & $1.56(0.67)$ & $0.25^{* * *}$ & $0.17^{* * *}$ \\
N2: Hostility & $1.39(0.51)$ & -0.02 & 0.00 \\
N3: Depression & $1.66(0.64)$ & $0.23^{* * *}$ & $0.12^{* *}$ \\
N4: Self-consciousness & $1.72(0.53)$ & -0.08 & -0.07 \\
N5: Impulsiveness & $1.84(0.48)$ & 0.03 & 0.02 \\
N6: Stress vulnerability & $1.30(0.46)$ & -0.05 & -0.05 \\
Education & $3.25(1.32)$ & & $-0.12^{* * *}$ \\
Sex (women, \%) & 64.6 & & $-0.08^{* * *}$ \\
Reduced activity/in bed (\%) & 11.3 & & $0.40^{* * *}$ \\
Medical condition (\%) & 40.9 & & $0.25^{* * *}$ \\
Functional impairment (\%) & 8.7 & & $0.46^{* * *}$ \\
\hline
\end{tabular}

Table 3. Model fitting results (best-fitting model shown in bold). A - Additive genetic effects; $\mathrm{C}$ - common environmental effects; E - non-shared environmental effects; AIC - Akaike's Information Criterion.

\begin{tabular}{lcccc}
\hline Model & $\begin{array}{c}-2 \log \\
\text { likelihood }\end{array}$ & df & $\Delta$-2LL $(\Delta \mathrm{df})$ & AIC \\
\hline 1. ACE (sex-specific parameters) & 14891.35 & 9018 & - & -3144.65 \\
2. AE (sex-specific parameters) & 14894.57 & 9030 & $3.22(12)$ & -3165.43 \\
3. AEC (equal standardized parameters across sex) & 14899.27 & 9027 & $7.92(9)$ & -3154.73 \\
4. AE (equal standardized parameters across sex) & 14900.23 & 9033 & $8.88(15)$ & $\mathbf{- 3 1 6 5 . 7 7}$ \\
\hline
\end{tabular}

and the MS symptoms index on the other, varied between 0.12 and 0.34 (all coefficients significant at the $\mathrm{p}<0.05$ level). N1: anxiety and N3: depression was found to be significant in a regression analysis including only the six facets as independent variables (Table 2 ). These associations remained significant when controlling for demographic and general health variables.

We next tested a set of trivariate Cholesky models including N1: anxiety, N3: depression, and the MS symptoms index. Table 3 shows the fit of the different models. Model 1 included A, C and E factors, and allowed estimates to vary across sex. Dropping shared environmental effects in model 2 did not result in a significant reduction in fit (i.e., $\Delta$-2LL $=3.22, \Delta \mathrm{df}=$ 12 , ns.) and produced a lower AIC value. Furthermore, model 3 and 4, in which the standardized path coefficients for both environmental and genetic effects were set equal for men and women, yielded additional improvements in fit, with the AE model having lowest AIC and thus designated as the best-fitting model.

Figure 1 shows the Cholesky parameters of the model. A genetic factor (A1) accounted for all the genetic variance in N1: anxiety, plus $37 \%$ of the total variance ( $81 \%$ of the genetic variance) in $\mathrm{N} 3$ : depression, and $15 \%(27 \%$ of the genetic variance $)$ in MS symptoms. A second genetic factor (A2), independent of A1, accounted for an additional $8 \%$ of the variance $(17 \%$ of the genetic variance) in N3: depression, but not significantly for variance in MS symptoms. The genetic factor A3 accounted for specific genetic variance $(38 \%)$, indicating that a large proportion of the genetic influence (i.e., $71 \%$ ) on MS symptoms was unique to the phenotype and not shared with the neuroticism facets. Based on the Cholesky model, heritability estimates were $0.47(95 \% \mathrm{CI}=0.42-0.52)$ for $\mathrm{N} 1$ : anxiety, $0.46(95 \% \mathrm{CI}=0.40-0.51)$ for $\mathrm{N} 3$ : depression, and 0.54 


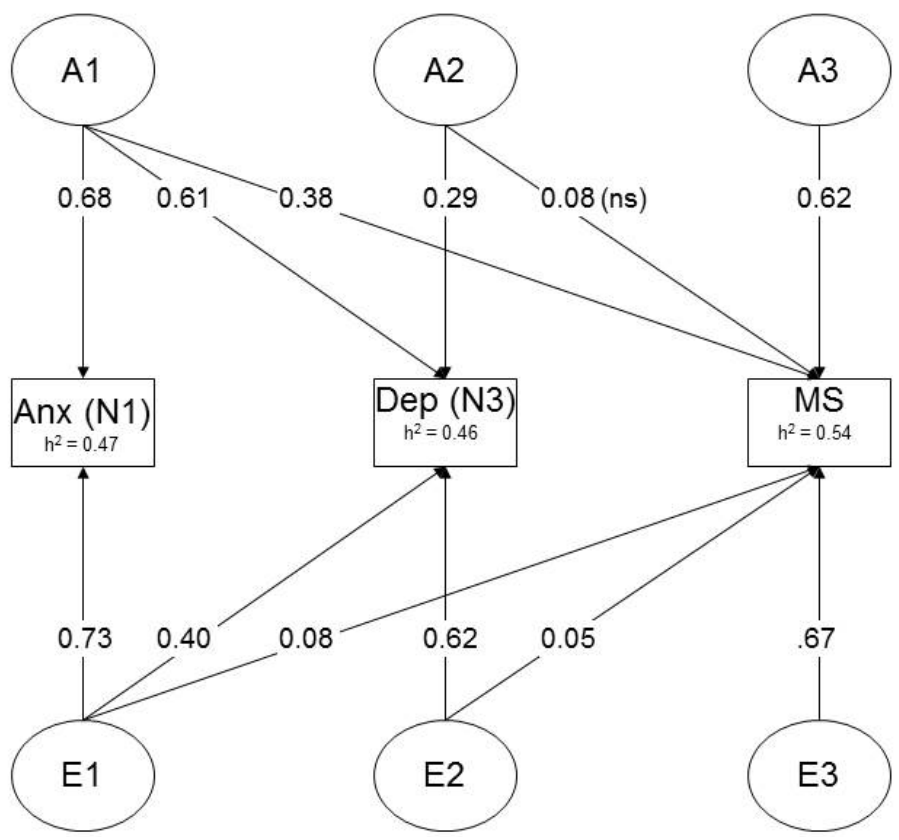

Figure 1. Parameter estimates for the trivariate AE Cholesky model showing covariation between N1: Anxiety, N3: depression, and MS symptoms. The model includes genetic (A1, A2, A3) and non-shared environmental (E1, E2, E3) sources. Estimates are standardized, thus indicating the percentage of variance accounted for when squared. Heritability $\left(\mathrm{h}^{2}\right)$ is shown for each variable. All parameters except the one marked $(\mathrm{ns})$ are significant $(\mathrm{p}<0.05)$.

(95\% CI=0.49-0.59) for MS symptoms. In contrast to the evident genetic association shared among the three phenotypes, environmental effects (i.e., the crosseffects of E1 and E2) were generally much smaller. While the cross-effect of E1 on N3: depression was non-trivial (i.e., 0.40), E1 and E2 together accounted for less than $1 \%$ of the variance in MS symptoms. However, the total non-shared environmental effects on each phenotype were substantial, accounting for a large amount of the variance (i.e. $53 \%$ for N1: anxiety, $54 \%$ for N3: depression, and $46 \%$ for MS symptoms).

The genetic correlations between MS symptoms on the one hand, and N1: Anxiety and N3: depression on the other, were both 0.52 (95\% CIs were $0.44-0.60$, and 0.43-0.60, respectively). The corresponding environmental correlations were also of equal magnitude, i.e., 0.12 (95\% CIs were 0.05-0.19, and 0.06-0.19, respectively).

\section{DISCUSSION}

Our study contributes to the research on psychological aspects of MS symptoms in primarily two ways. First, the relationship of neuroticism facets with MS symptoms was investigated, for the first time we assume, in a genetically informative design. Second, a composite MS index, based on previous psychometric research $(32,33)$ and psychometric results obtained in the present sample, was used in regression analyses and biometric modeling. In congruence with the findings reported by Williams et al. (41), significant associations between symptoms at different anatomical sites were found, indicating that a single, common factor accounts for much of the liability to report MS symptoms.

As expected, our results showed that MS symptoms were commonly reported in this population-based sample of middle-aged twins. Furthermore, demographic and general health variables were significantly associated with MS symptoms when entered simultaneously as independent variables in multiple regression analyses. Of note, women evidenced a higher MS complaint level than men, even after adjustment for education, general health indices, and neuroticism facets. This finding is in keeping with accumulated research on sex differences in chronic pain conditions, suggesting the existence of a female-specific pain psychobiology (9). Of significance, however, the bestfitting biometric model did not include any sexspecific parameters. Thus, while there are systematic sex differences in pain symptom levels (9) and neuroticism scores (26), the underlying genetic and environmental variance-covariance structures do not appear markedly sex-specific.

The heritability estimates of MS symptoms and neuroticism facets were broadly consistent with those reported in previous studies $(4,42,43)$. Cholesky modeling furthermore revealed significant and nontrivial genetic cross-effects from N1: anxiety to N3: depression and MS symptoms, but not from N3: depression to MS symptoms. However, the genetic correlations between N1: anxiety and N3: depression on the one hand, and MS symptoms on the other, were substantial (i.e., 0.52), as was the genetic correlation between the two $\mathrm{N}$ facets (i.e., 0.90). Hence, which of these facets are put in the first position in the Cholesky 
model is more or less arbitrary, the essential finding being that the phenotypic correlations are significantly influenced by common genetic factors. In the model shown in Figure 1, a strongly influential common genetic factor was identified, accounting for nearly $50 \%$ of the total variance in N1: anxiety and around $81 \%$ and $27 \%$ of the genetic variance in N3: depression and MS symptoms, respectively. This factor may reflect a general susceptibility to psychological and somatic distress (44), which is a core characteristic of the neuroticism trait (i.e., depression, hostility, and stress/ anxiety related psychophysiological activation).

Accumulated research across decades has documented the consistent association between neuroticism and the presence of somatic complaints and illnesses (44, 45). Researchers have offered a variety of explanations, including both spurious and causal relationships, when describing these neuroticism-health associations. A first possibility is that neuroticism is associated with a greater internal self-focus and a tendency to worry/ rumination, resulting in an exaggerated preoccupation with physical symptoms and therefore a greater symptom report bias (44). However, several largescale longitudinal studies have documented that initial neuroticism levels are predictive of somatic and psychiatric morbidity assessed several decades later $(23,46)$. Furthermore, the employment of various statistical control procedures, such as adjustment for simultaneous neuroticism scores, indicates that such longitudinal findings are unlikely to be the result of manifest psychiatric disorder or reporting bias. Of significance, individuals with higher neuroticism levels are vulnerable to a broad range of mental disorders (i.e., anxiety, depression, schizophrenia, personality disorders), higher levels of comorbidity, medically unexplained somatic symptoms, and general health problems, including cardiovascular disease, irritable bowel syndrome, and chronic widespread pain $(23,47)$. For these reasons, neuroticism may not reflect just a symptom perception and report bias, but is in all likelihood also linked to symptom generating neurobiological processes. The significant genetic correlations that emerged in the present study indicate that a common genetic vulnerability may explain the neuroticism-MS symptoms association. In light of the research referred to above, it appears unlikely that common genes and mechanisms are restricted to exert their effects solely on symptom perception and symptom reporting processes.

A second explanation, related to the discussion of the symptom perception hypothesis, maintains that chronic emotional instability and psychophysiological activation trigger off pain enhancing, inflammatory and other physiological processes, resulting in wear and tear in the body, increased symptom levels, and illness liability. In a longitudinal twin study, Charles et al. (23) showed that the likelihood of having a somatic conditions (e.g., chronic fatigue syndrome, ulcers, coronary heart disease, chronic widespread pain) was related to higher levels of neuroticism assessed 25 years earlier. Neuroticism exerted the strongest effects for conditions involving systemic pain. Many researchers $(48,49)$ maintain that higher levels of neuroticism and negative affect symptoms such as anxiety and depression may disturb normal pain responding and over time cause pain sensitization and other disabling effects. As documented in numerous pain experiments, repeated exposure to noxious stimulation may lead to amplified pain response (sensitization) or diminished pain response (habituation), and these phenomena seem to involve both peripheral and central processes (50). Recently, Nakamura et al. (51) showed that participants in an experimental pain study on average showed amplification (sensitization) of pain report over trials. Of note, the personality traits neuroticism and conscientiousness contributed to the variance in pain amplification. However, the statistical effects were rather modest, and the authors were careful to point out that their results do not indicate that personality traits explain clinical pain conditions characterized by central sensitization. Moreover, while personality traits and negative affect symptoms appear, depending on pain modality, essentially unrelated to or only weakly related to sensory pain sensitivity (28), they are evidently more strongly associated with the affective-motivational dimensions of pain in both clinical and non-clinical populations $(52,53)$. For example, in a study of fibromyalgia patients (54), depressive symptoms and anxiety correlated with pain catastrophizing and subjective rating of general health, but did not correlate with pressure pain sensitivity or cerebral processing of pain as assessed by functional magnetic resonance imaging (fMRI).

Altered immune function should also be considered as a possible mechanism underlying neuroticism and somatic complaints. One recent and potentially important insight from psychoneuroimmunological research is the discovery that components of the immune system that mediate inflammation may be intimately involved in negative affect symptoms (55). Normally, inflammatory activity is provoked by physical stimuli such as infections or injuries. There is now substantial evidence that stress/negative affect can trigger significant increases in inflammatory activity (i.e., in the absence of physical injury/infection). Amplified inflammation can in turn influence pain perception and induce symptoms such as fatigue, depressive symptoms and social-behavioral withdrawal (56). Notably, some studies have reported significant associations between personality traits and inflammation markers. A population-based study, including nearly 5000 participants, showed that high neuroticism and low conscientiousness were both associated with higher levels of the pro-inflammatory cytokine interleukin-6 (IL-6) (57). However, the associations were weak (i.e., correlations in the 0.04-0.07 range), and some studies have failed to find an association between neuroticism and IL-6 (58).

A third general explanation for the neuroticism- 
health relationship is that either the somatic condition or its treatment may give rise to rather than result from negative affect symptoms and elevated neuroticism. Generally, life events including acute and chronic illnesses are unrelated to or only weakly related to neuroticism or the other personality domains within the five-factor framework (27). It is worth mentioning, however, that a recent longitudinal study of long-time consequences of chronic, serious diseases such as cancer, stroke and heart disease on personality traits has indeed documented significant effects (59). However, the effects were weak to moderate (i.e., T score changes on the order of $0.25-0.44)$, and the agreeableness trait was unaffected by chronic disease. As shown in the Cholesky analysis in the present stu$\mathrm{dy}$, the environmental influences were largely specific to each phenotype. These model parameters reflect, besides measurement error, environmental factors unique to the trait and to the individual, such as occupational risk factors, stressful life events, injuries and illnesses. Obviously, without further information it is uncertain to what extent such factors are reflected in significant $\mathrm{E}$ parameters in the present model.

Of the potentially important individual-specific environmental effects on MS conditions, psychosocial working conditions and physical workloads should be mentioned in particular (8). In a meta-analysis, Hauke et al. (60) concluded that working conditions characterized by low social support, high job demands, low job control, low decision authority, and low job satisfaction were associated with risk for MS pain in the low back, the neck/shoulder, and the upper extremities. However, as pointed out by several authors $(7,60)$, much research in this area is to a large extent inconclusive and is often focused on a circumscribed body region, such a neck/shoulder or low back pain. For example, Clausen et al. (7) hold that most studies have failed to adjust for physical workload or different types of negative affectivity, both of which may be important confounders. In their own prospective study, Clausen et al. (7) showed that psychosocial working conditions such as emotional demands, role conflicts and work influence predicted low back pain for between 1 and 30 days in the past year at follow-up after adjustment for sociodemographic variables, health behaviors, and physical workload. However, most associations became statistically insignificant when adjusted for depressive symptoms at baseline. The authors maintain that depressive symptoms will cause over-reporting of both adverse psychosocial working conditions and MS pain, which implies that the observed associations between independent and dependent variables may be spurious. Similar results were reported in a study (61) of anxiety/depression symptoms in shift workers, using neuroticism as the main control variable. In a series of simple regression models, most of the psychosocial work factors were significantly related to anxiety/ depression symptoms, controlling for age, sex, and education level. When neuroticism was included in the regression models, several of these associations were no longer significant, and due to the strong statistical effect of neuroticism the amount of explained variance in the dependent variables increased substantially. Thus, one important implication of these studies is that the perception and appraisal of environmental characteristics are inherently linked to stable and heritable personality traits.

The present study has notable strengths such as using a genetically informative sample of twins that can be considered representative of a large segment of the Norwegian general population. Additionally, all the measurement instruments have established psychometric properties and have been used in previous epidemiological research and in twin studies $(6,25)$. Nevertheless, some limitations should also be noted. First, our assessment was based on self-reports, without physical examinations or other objective data. Thus, clinical conditions that may cause MS symptoms were not assessed, and the results may not be directly comparable to those based on clinical samples. For example, in a twin study of the role of disc degeneration (assessed by lumbar MRI) in low back pain (62), significant genetic correlations were found for disc height narrowing and different definitions of back pain, such as duration of the worst back pain episode and disability in the previous year from back pain. The heritability estimate was 0.51 for disc height and in the 0.31-0.37 range for the various definitions of low back pain. However, as noted by Williams et al. (41) and others, there is often a lack of correspondence between (subjective) clinical pain and objective findings such as radiographic change. It can furthermore be argued that pain reporting is significant and valid in its own right as it represents the symptoms with which patients present to their physicians. Lastly, the genetic and environmental structure underlying subjective vs. objective aspects of MS symptoms are evidently very different so one should not be used as a substitute for the other (41).

Second, the data were obtained on only one occasion. Life-course epidemiology points to the need for longitudinal research designs to investigate the course of both somatic and psychiatric conditions. Crosssectional study designs tend to mix single-episode cases with recurrent and chronic cases, which are known to differ with regard to the extent of comorbidity, disability, and possibly etiology (63). It should be emphasized, however, that the relationship between neuroticism, negative affect symptoms, and somatic complaints appears highly robust and has been demonstrated across developmental phases, from childhood to old age (45). Nevertheless, there is an obvious need for longitudinal, genetically informative studies in this research area.

A third limitation may be the conceptualization of neuroticism facets and subjective somatic symptoms as distinct phenotypes, given the relative lack of empirical data to support a clear separation (24). For example, 
Watson and Pennebaker (44), in their pioneering studies, maintained that the systematic associations among numerous negative affect and somatic complaints measures were due to an underlying 'somatopsychic distress factor'. Additionally, various symptom clusters such as functional somatic syndromes (16), together with anxiety and depression, may be considered part of a broader spectrum of internalizing disorders (64). Still, comprehensive factor analytic studies indicate a clear separation (65-67). Of particular note, our own findings as well as previous research $(16,24)$ have demonstrated that while neuroticism, anxiety/depression symptoms, and somatic complaints are related, they are also distinct in terms of underlying genetic and environmental factors.

In summary, our results indicate that a substantial part of the variance in symptom reporting at different anatomical sites can be accounted for by a single underlying factor. This generalized symptom suscep- tibility is furthermore phenotypically linked to the neuroticism facets N1: anxiety and N3: depression, and to sex, education level and indices of general health condition. Cholesky modeling indicated substantial unique genetic and non-shared environmental influences on each phenotype, whereas genetic sources accounted for most of the associations among the three phenotypes. How these findings should be accounted for in terms of underlying genetic architecture and psychobiological mechanisms, must still be regarded as an open question. There are probably several causes and processes involved such as central pain modulation and inflammatory activity. The clinical implications of the relationship between pain, anxiety and depression have been emphasized by many authors (19). The present study contributes to this discussion by showing that the neuroticism trait facets $\mathrm{N} 1$ : anxiety and N3: depression are genetically linked to MS symptoms.

\section{REFERENCES}

1. Diatchenko L, Fillingim RB, Smith SB, Maixner W. The phenotypic and genetic signatures of common musculoskeletal pain conditions. Nature Rev Rheumatol 2013;9(6):340-50.

2. Bergman S, Herrstrom P, Hogstrom K, Petersson IF, Svensson B, Jacobsson LT. Chronic musculoskeletal pain, prevalence rates, and sociodemographic associations in a Swedish population study. J Rheumatol 2001; 28(6):1369-77.

3. Cimmino MA, Ferrone C, Cutolo M. Epidemiology of chronic musculoskeletal pain. Best Pract Res Clin Rheumatol 2011;25(2):173-83.

4. Nyman T, Mulder M, Iliadou A, Svartengren M, Wiktorin C. High heritability for concurrent low back and neck-shoulder pain: a study of twins. Spine 2011;36(22):E1469-76.

5. Kato K, Sullivan PF, Evengard B, Pedersen NL. Chronic widespread pain and its comorbidities: a populationbased study. Arch Intern Med 2006;166(15):1649-54.

6. Vassend O, Skrondal A. The role of negative affectivity in self-assessment of health: A structural equation approach. J Health Psychol 1999;4(4):465-82.

7. Clausen T, Andersen LL, Holtermann A, Jorgensen AFB, Aust B, Rugulies R. Do self-reported psychosocial working conditions predict low back pain after adjustment for both physical work load and depressive symptoms? A prospective study among female eldercare workers. Occup Environ Med 2013;70(8):538-44.

8. Bugajska J, Zolnierczyk-Zreda D, Jedryka-Goral A, Gasik R, Hildt-Ciupinska K, Malinska M, et al. Psychological factors at work and musculoskeletal disorders: a one year prospective study. Rheumatol Int 2013; 33(12):2975-83.

9. Mogil JS. Sex differences in pain and pain inhibition: multiple explanations of a controversial phenomenon. Nature Rev Neurosci 2012;13(12):859-66.

10. Kamaleri Y, Natvig B, Ihlebaek CM, Benth JS, Bruusgaard D. Change in the number of musculoskeletal pain sites: A 14-year prospective study. Pain 2009;141(1-2):25-30.

11. Hoftun GB, Romundstad PR, Zwart JA, Rygg M. Chronic idiopathic pain in adolescence - high prevalence and disability: The young HUNT study 2008. Pain 2011;152(10):2259-66.

12. Jussila L, Paananen M, Nayha S, Taimela S, Tammelin T, Auvinen J, et al. Psychosocial and lifestyle correlates of musculoskeletal pain patterns in adolescence: A 2-year follow-up study. Eur J Pain 2014;18(1):139-46.

13. Nielsen CS, Knudsen GP, Steingrimsdottir OA. Twin studies of pain. Clin Genet 2012;82(4):331-40.

14. Fejer R, Hartvigsen J, Kyvik KO. Heritability of neck pain: a population-based study of 33,794 Danish twins. Rheumatology 2006;45(5):589-94.

15. Hartvigsen J, Petersen HC, Frederiksen H, Christensen K. Small effect of genetic factors on neck pain in old age: a study of 2,108 Danish twins 70 years of age and older. Spine 2005;30(2):206-8.

16. Kato K, Sullivan PF, Evengard B, Pedersen NL. A population-based twin study of functional somatic syndromes. Psychol Med 2009;39(3):497-505.

17. Krishnan KRR, France RD, Pelton S, Mccann UD, Davidson J, Urban BJ. Chronic pain and depression 2. Symptoms of anxiety in chronic low-back pain patients and their relationship to subtypes of depression. Pain 1985;22(3):289-94. 
18. McWilliams LA, Cox BJ, Enns MW. Mood and anxiety disorders associated with chronic pain: an examination in a nationally representative sample. Pain 2003;106(1-2):127-33.

19. Bair MJ, Wu J, Damush TM, Sutherland JM, Kroenke K. Association of depression and anxiety alone and in combination with chronic musculoskeletal pain in primary care patients. Psychosom Med 2008;70(8):890-7.

20. Hartvigsen J, Frederiksen H, Christensen K. Physical and mental function and incident low back pain in seniors: a population-based two-year prospective study of 1387 Danish twins aged 70 to 100 years. Spine 2006;31(14):1628-32.

21. Lahey BB. Public health significance of neuroticism. Am Psychol 2009;64(4):241-56.

22. Hettema JM, Neale MC, Myers JM, Prescott CA, Kendler KS. A population-based twin study of the relationship between neuroticism and internalizing disorders. Am J Psychiatry 2006;163(5):857-64.

23. Charles ST, Gatz M, Kato K, Pedersen NL. Physical health 25 years later: The predictive ability of neuroticism. Health Psychol 2008;27(3):369-78.

24. Hansell NK, Wright MJ, Medland SE, Davenport TA, Wray NR, Martin NG, et al. Genetic co-morbidity between neuroticism, anxiety/depression and somatic distress in a population sample of adolescent and young adult twins. Psychol Med 2012;42(6):1249-60.

25. Vassend O, Roysamb E, Nielsen CS. Neuroticism and self-reported somatic health: A twin study. Psychol Health 2012;27(1):1-12.

26. Costa PT, McCrae RR. Revised NEO Personality Inventory (NEO-PI-R) and NEO Five-Factor Inventory (NEO-FFI). Odessa, FL: Psychological Assessment Resources, 1992.

27. McCrae RR, Costa Jr PT. Personality in adulthood. A five-factor theory perspective. New York: Guilford Press, 2006.

28. Vassend O, Roysamb E, Nielsen CS. Five-factor personality traits and pain sensitivity: a twin study. Pain 2013;154(5):722-8.

29. Harris JR, Magnus P, Tambs K. The Norwegian Institute of Public Health twin program of research: an update. Twin Res Hum Genet 2006;9(6):858-64.

30. Magnus P, Berg K, Nance WE. Predicting zygosity in Norwegian twin pairs born 1915-1960. Clin Genet 1983;24(2):103-12.

31. Martinsen Ø, Nordvik H, Østbø LE. NEO-PI-R. Oslo: Gyldendal, 2003.

32. Brähler E, Scheer J. Der Giessener Beschwerdebogen. Handbuch [Manual of the Giessen Symptom Checklist]. Bern: Hans Huber, 1983.

33. Vassend O, Lian L, Andersen HT. Norwegian versions of the NEO-Personality Inventory, Symptom Checklist 90 Revised, and Giessen Subjective Complaints List: I. Tidsskrift for Norsk Psykologforening ((Journal of the Norwegian Psychological Association) 1992;29(12):1150-60.

34. Moum T. Self-assessed health among Norwegian adults. Soc Sci Med 1992;35(7):935-47.

35. Carlin JB, Gurrin LC, Sterne JAC, Morley R, Dwyer T. Regression models for twin studies: a critical review. Int J Epidemiol 2005;34(5):1089-99.

36. Neale MC, Hunter MD, Pritikin JN, Zahery M, Brick TR, Kirkpatrick RM, et al. OpenMx 2.0: Extended structural equation and statistical modeling. Psychometrika 2016;81(2):535-49.

37. Carey G. Cholesky problems. Behav Genet 2005;35(5):653-65.

38. Loehlin JC. The Cholesky approach: A cautionary note. Behav Genet 1996;26(1):65-9.

39. Neale MC, Roysamb E, Jacobson K. Multivariate genetic analysis of sex limitation and $\mathrm{G} x \mathrm{E}$ interaction. Twin Res Hum Genet 2006;9(4):481-9.

40. Akaike H. Factor-analysis and AIC. Psychometrika 1987;52(3):317-32.

41. Williams FMK, Spector TD, MacGregor AJ. Pain reporting at different body sites is explained by a single underlying genetic factor. Rheumatology 2010;49(9):1753-5.

42. Jang KL, McCrae RR, Angleitner A, Riemann R, Livesley WJ. Heritability of facet-level traits in a crosscultural twin sample: Support for a hierarchical model of personality. J Pers Soc Psychol 1998;74(6):1556-65.

43. Reichborn-Kjennerud T, Stoltenberg C, Tambs K, Roysamb E, Kringlen E, Torgersen S, et al. Back-neck pain and symptoms of anxiety and depression: a population-based twin study. Psychol Med 2002;32(6):1009-20.

44. Watson D, Pennebaker JW. Health complaints, stress, and distress - Exploring the central role of negative affectivity. Psychol Rev 1989;96(2):234-54.

45. Friedman HS, Kern ML. Personality, well-being, and health. Annu Rev Psychol 2014;65:719-42.

46. Neeleman J, Sytema S, Wadsworth M. Propensity to psychiatric and somatic ill-health: evidence from a birth cohort. Psychol Med 2002;32(5):793-803.

47. Cuijpers P, Smit F, Penninx BW, de Graaf R, ten Have M, Beekman AT. Economic costs of neuroticism: a population-based study. Arch Gen Psychiatry 2010;67(10):1086-93.

48. Wilhelmsen I. Biological sensitisation and psychological amplification: Gateways to subjective health complaints and somatoform disorders. Psychoneuroendocrinology 2005;30(10):990-5.

49. Janssen SA. Negative affect and sensitization to pain. Scand J Psychol 2002;43(2):131-7. 
50. Woolf CJ. Central sensitization: Implications for the diagnosis and treatment of pain. Pain 2011;152(3):S2-15.

51. Nakamura Y, Donaldson GW, Okifuji A. Personality, anxiety, and individual variation in psychophysiological habituation and sensitization to painful stimuli. J Pain Relief 2014;3(3):1-9.

52. Giesecke T, Gracely RH, Williams DA, Geisser ME, Petzke FW, Clauw DJ. The relationship between depression, clinical pain, and experimental pain in a chronic pain cohort. Arthritis Rheum 2005;52(5):1577-84.

53. Lee JE, Watson D, Law LAF. Lower-order pain-related constructs are more predictive of cold pressor pain ratings than higher-order personality traits. J Pain 2010;11(7):681-91.

54. Jensen KB, Petzke F, Carville S, Fransson P, Marcus H, Williams SCR, et al. Anxiety and depressive symptoms in fibromyalgia are related to poor perception of health but not to pain sensitivity or cerebral processing of pain. Arthritis Rheum 2010;62(11):3488-95.

55. Slavich GM, Irwin MR. From stress to inflammation and major depressive disorder: A social signal transduction theory of depression. Psychol Bull 2014;140(3):774-815.

56. Dimsdale JE, Dantzer R. A biological substrate for somatoform disorders: Importance of pathophysiology. Psychosom Med 2007;69(9):850-4.

57. Sutin AR, Terracciano A, Deiana B, Naitza S, Ferrucci L, Uda M, et al. High neuroticism and low conscientiousness are associated with interleukin-6. Psychol Med 2010;40(9):1485-93.

58. Chapman BP, Khan A, Harper M, Stockman D, Fiscella K, Walton J, et al. Gender, race/ethnicity, personality, and interleukin-6 in urban primary care patients. Brain Behav Immun 2009;23(5):636-42.

59. Jokela M, Hakulinen C, Singh-Manoux A, Kivimaki M. Personality change associated with chronic diseases: pooled analysis of four prospective cohort studies. Psychol Med 2014;44(12):2629-40.

60. Hauke A, Flintrop J, Brun E, Rugulies R. The impact of work-related psychosocial stressors on the onset of musculoskeletal disorders in specific body regions: A review and meta-analysis of 54 longitudinal studies. Work Stress 2011;25(3):243-56.

61. Berthelsen M, Pallesen S, Bjorvatn B, Knardahl S. Shift schedules, work factors, and mental health among onshore and offshore workers in the Norwegian petroleum industry. Ind Health 2015;53(3):280-92.

62. Battie MC, Videman T, Levalahti E, Gill K, Kaprio J. Heritability of low back pain and the role of disc degeneration. Pain 2007;131(3):272-80.

63. Caspi A, Houts RM, Belsky DW, Goldman-Mellor SJ, Harrington H, Israel S, et al. The p factor: One general psychopathology factor in the structure of psychiatric disorders? Clin Psychol Sci 2014;2(2):119-37.

64. Krueger RF, Markon KE. Understanding psychopathology: Melding behavior genetics, personality, and quantitative psychology to develop an empirically based model. Curr Dir Psychol Sci 2006;15(3):113-7.

65. Gillespie N, Kirk KM, Heath AC, Martin NG, Hickie I. Somatic distress as a distinct psychological dimension. Soc Psychiatry Psychiatr Epidemiol 1999;34(9):451-8.

66. Kotov R, Ruggero CJ, Krueger RF, Watson D, Yuan QL, Zimmerman M. New dimensions in the quantitative classification of mental illness. Arch Gen Psychiatry 2011;68(10):1003-11.

67. Simms LJ, Prisciandaro JJ, Krueger RF, Goldberg DP. The structure of depression, anxiety and somatic symptoms in primary care. Psychol Med 2012;42(1):15-28. 